ganglia, and well-defined lesions). In children $<10$ years, Barkhof criteria had a higher sensitivity than KIDMUS criteria, but lower than in older children. Mean time to conversion to MS after a CIS was 17.7 months (range 2-75 months); after a PCIS attack, the mean time to MS diagnosis was 24 months (range 2-79 months). (Neuteboom RF, Boon M, Berrevoets CEC, et al. Prognostic factors after a first attack of inflammatory CNS demyelination in children. Neurology Sept 2008;71:967-973). (Reprints: Dr RQ Hintzen, Department of Neurology, MS centre ErasMS, Erasmus MC, Rotterdam. E-mail: r.hintzen(äerasmusmc.nl).

COMMENT. Barkhof and KIDMUS MRI criteria have a high specificity and are risk factors for conversion to MS in children with a first demyelinating attack, but their sensitivity is poor, especially at age $<10$ years. Over a mean period of observation of 54 months (range 5-201 months), 37 children (31\%) developed MS. In this study children with initial monofocal symptoms were more likely to have MS (43\%) compared to those with polyfocal features $(21 \%)$. In contrast, $17 \%$ of children with an initial ADEM-like presentation were diagnosed with MS at follow-up (Banwell BL. Editorial. Neurology 2008;71:962-963).

\title{
SIGNIFICANCE OF MRI PERIVASCULAR SPACES IN MS
}

The role of perivascular Virchow-Robin spaces was investigated in 45 multiple sclerosis (MS) patients and 30 healthy controls, in a study at Charite-Universitaetsmedizin Berlin, and Goethe University, Frankfurt. Virchow-Robin spaces (VRS) that surround small blood vessels as they penetrate brain parenchyma were identified in the same number of MS patients as healthy controls. However, the VRS were significantly larger in volume in MS $(\mathrm{p}=0.004)$. This difference was not explained by brain atrophy but at follow-up, a significant increase in VRS volume was correlated with contrast-enhancing lesions, indicative of inflammation. VRS volume increase may be supportive of inflammatory demyelination in the brain. (Wuerfel J, Haertle M, Waiczies H, et al. Perivascular spaces - MRI marker of inflammatory activity in the brain? Brain Sept 2008;131(9):2332-2340). (Respond: Prof Dr F Zipp, Scientific Director of the Cecilie Vogt Clinic for Neurology in the HKBB, Chariteplatz 1, Charite-Universitaetsmedizin Berlin, 10117 Berlin, Germany.

E-mail:frauke.zipp@charite.de).

COMMENT. The authors interpret the prominence of Virschow-Robin spaces in the brain of MS patients as a sign of inflammation, not of age, and VRS are recognized for their potential as modulators of immune responses.

The prevalence and clinical significance of dilated VRS in childhood was investigated by MRI in 1,250 patients, during 12 consecutive months at Children's Medical Center, Dallas, TX. (Rollins NK et al. Radiology 1993;189:53-57). Of 37 patients with prominent VRS, 12 had severe headache, 17 had moderate or severe delay in development, and 18 had serious behavioral or psychiatric problems. The association of these symptoms and dilated VRS was significant $(\mathrm{p}<0.001)$.

In a retrospective review of $816 \mathrm{MR}$ scans, 314 had large VRS in a study at New York Hospital, NY (Heir LA et al. AJNR 1989;10:929-936). Of patient variables studied statistically, that included age, gender, incidental white matter lesions, infarction, dementia, hypertension, and atrophy, only age was significantly correlated with large VRS. 
Kwee RM et al in the Netherlands review the significance of dilated VRS (Radiographics 2007;27:1071-1086). The three characteristic locations for dilated VRS are lenticulostriate arteries, perforating medullary arteries, and the midbrain. Occasionally, VR spaces become very large and even cause mass effect. The signal intensity is identical to that of CSF. They should be differentiated from lacunar infarctions, periventricular leukomalacia, multiple sclerosis, arachnoid cysts, cystic neoplasms, and neurocysticercosis.

\section{DEVELOPMENTAL DISORDERS}

\section{RISKS OF PLAGIOCEPHALY AND TORTICOLLIS IN NEWBORNS}

The incidence and characteristics of torticollis, plagiocephaly and facial asymmetry were evaluated prospectively by photographic analysis in 102 healthy newborn infants in a study at University of California, San Diego. Asymmetries of craniofacial development affecting $73 \%$ of newborns included torticollis in $16 \%$, asymmetrical mandible in $13 \%$, facial asymmetry in $42 \%$, and asymmetry of the head in $61 \%$. Malposition of head and neck in utero for 6 weeks or more before delivery was associated with torticollis; larger babies, birth trauma and long labor with facial asymmetry; and birth trauma with plagiocephaly. Identification of affected newborns should prompt positioning recommendations to prevent secondary craniofacial deformities. (Stellwagen L, Hubbard E, Chambers C, Jones KL. Torticollis, facial asymmetry and plagiocephaly in normal newborns. Arch Dis Child Oct 2008;93:827-831). (Respond: Dr L Stellwagen, Mail Code 8774, Division of Neonatology, University of California, San Diego, Medical Center, 200 West Arbor Drive, San Diego, CA 92103. E-mail: Istellwagen(aucsd.edu).

COMMENT. Early detection of torticollis by neck range of motion assessment at birth may prompt physiotherapy and head positioning that will prevent posterior plagiocephaly.

\section{DEVELOPMENTAL OUTCOME OF PRETERM INFANTS WITH SEVERE INTRAVENTRICULAR HEMORRHAGE}

Of 214 preterm infants studied at Wilhelmina Children's Hospital, Utrecht, the Netherlands, $94(44 \%)$ had a grade III intraventricular hemorrhage (IVH), and $120(56 \%)$ had a grade IV hemorrhage. Post-hemorrhagic ventricular dilatation (PHVD) developed significantly more often in survivors with grade III hemorrhage $(78 \%)$ than in infants with grade IV hemorrhage $(53 \%)(\mathrm{p}=0.002)$. Cerebral palsy developed in $7.4 \%$ of the surviving grade III group, compared with $48.7 \%$ with a grade IV hemorrhage $(p<0.001)$. The mean developmental quotient (DQ) was 99 in grade III group and 95 in the grade IV group at 24 months corrected age. The DQ was significantly lower in infants with cerebral palsy, irrespective of severity of IVH. (Brouwer A, Groenendaal F, van Haastert I-L, et al. J Pediatr May 2008;152:648-654). (Reprints: Linda S de Vries MD PhD, Department of Neonatology, Wilhelmina Children's Hospital, Utrecht, The Netherlands. E-mail: LS.deV'ries'(a umcutrecht.11l).

COMMENT. Outcome was better than expected in preterm infants with severe IVH. 